\title{
Numerical Study of the Effect of Orientation Angles on Combined Convection in a Cavity Contain Rotation Cylinder
}

\author{
Sana.M.Shrama ${ }^{1}$, Falah A. Abood ${ }^{2}$, Sana.J.Yaseen ${ }^{3}$, Amani J. Majeed ${ }^{4 *}$ \\ \{sanamech77@gmail.com,dr.falah.abood@gmail.com, \\ sana.yaseen@uobasrah.edu.iq, ammani.majeed@uobasrah.edu.iq\} \\ Mechanical Engineering department, Basrah University / Basrah, Iraq ${ }^{1,2,3}$ \\ Petroleum Engineering department, Basrah University / Basrah, Iraq ${ }^{4 *}$
}

\begin{abstract}
This study examines the influence of a rotating cylinder on heat transmitted by mixed convection in an air-filled square space. The appropriate mathematical models for continuous, incompressible, 2-dimensional, laminar flows with Boussinseq's approach are solved numerically, where constant fluid properties have been used. The most important parameters examined are: the angular velocity $(\Omega=$ 0 - 1000) and the angle of inclination $\left(\Theta=0-90^{\circ}\right)$, the study has been carried out with the Rayleigh number $\left(\mathrm{Ra}=10^{4}\right)$, the Prandtl number $(\operatorname{Pr}=0.7)$. The results show that the streamlines are affected by the angular velocity, where the forced convection becomes dominant at high values of angular velocity. The rotation of the cylinder has an effect on the heat exchange between the walls of the cavity and the fluid. Finally the average Nusselt number is increased with increases of angular velocity and decreases as inclination angles change from horizontal to vertical position. A good agreement has been achieved by comparing the results of this work with other previous work.Keywords: Inclined square cavity, rotating cylinder, mixed convection, numerical method.
\end{abstract}

\section{Nomenclature}

$\mathrm{Cp}$ constant pressure specific heat

g gravitational acceleration

$\mathrm{H}$ height (width) of the enclosure

$\mathrm{k}$ thermal conductivity

$\mathrm{Nu} \quad$ Nusselt number

p pressure

Pr Prandtl number

$\mathrm{r}$ radius

$\mathrm{R}$ radius at the surface of the cylinder

$\mathrm{Ra} \quad$ Rayleigh number

$\mathrm{Ri}$ Richardson number

$\mathrm{T}$ temperature

$\mathrm{u}, \mathrm{v}$ Cartesian velocity components

$\mathrm{x}, \mathrm{y}$ Cartesian co-ordinates

\section{Greek symbols}

$\alpha \quad$ thermal diffusivity

$\mu \quad$ dynamic viscosity

$v \quad$ kinematic viscosity

$\rho$ density

$\psi \quad$ streamfunction

$\Omega \quad$ angular rotational velocity

$\varphi \quad$ inclination angle 


\section{Introduction}

Natural convection in enclosed spaces has been widely analyzed, because of its use in cooling electronic equipment, nuclear and chemical reactors, heat exchangers, and other applications [1]. The buoyancy force and partially heated enclosure walls cause natural convection, while the rotation of cylinder causes forced convection. Both of them influence the resulting flow and create mixed convection. The direction of rotation matching with forced and natural convection, therefore it is results in the strengthening or in the weakening of the convective flow. The free convection of a porous medium in a vertical square cavity was studied numerically [1], the $\mathrm{Nu}$ was increased with $\mathrm{Ra}$. The mixed convection is studied in a square cavity with a concentric rotating cylinder studied numerically [2]. The impact of the cylinder's radius, rotational velocity, thermal capacity, and thermal conductivity were investigated. It can be noticed that the cylinder's size has a significant impact on the resulting flow and heat transfer process. Numerical simulations of $2 \mathrm{D}$, laminar mixed convection, and steady-state in a sinusoidal corrugated vented cavity with a heat conducting cylinder were performed [3]. Richardson and Reynolds numbers are taken in the ranges $0-10$ and 50-200, respectively; thermal conductivity ratio changed from 0.2 to 10 . The results showed that the average Nusselt number increased, and the average temperature of the inside corrugated cavity decreased with increasing the diameter of the cylinder. A rotating circular with mixed convection inside a square cavity at different vertical places and different Richardson number was calculated numerically. The study showed that the temperature distribution and the flow field within the enclosure relied powerfully on the Richardson numbers and the location of the rotating cylinder, the great value of heat transfer rate obtained near the lowest wall of the enclosure [4]. The effect of mixed convection on triangular enclosure contains a rotating single or multi cylinders investigated numerically [5], three cases were examined: a single rotating cylinder, three rotating cylinders in the same and in different directions. The most important parameters examined were Rayleigh number with range of $102-105$, angular velocity with range of $(\Omega=$ 0 - 1000) (for both cases). The result show that the mean $\mathrm{Nu}$ number for the single or multiple cylinders increased according to increasing $\mathrm{Ra}$, and $\Omega$. Also, the mean $\mathrm{Nu}$ number of a single cylinder was greater than that of multiple cylinders.

Mixed convection of a heated concentration cylinder with the enclosure studied numerically. The effect of different values of Lewis and Richardson numbers and buoyancy ratio were studied. It can be observed at the same Ri number the value of average Sherwood number raised as Lewis number growths. The mean $\mathrm{Nu}$ number about the cylinder reduced incrementally with the increase in buoyancy ratio at $\mathrm{Ri}$ number $1 / 40.01$, the for all Lewis numbers except at $1 / 40.1$ [6]. The effect of surface roughness on the turbulent flow through a rotating cylinder was calculated numerically [7]. The study was performed at three values of Reynolds numbers $(5 \times 105,106$ and 5x106) and fluctuation rates (1,2 and 3). The study showed that the drag coefficient improved with increasing surface roughness and rate of rotation, but decreased with increasing Reynolds number.

The heat transfer of a rotating heated cylinder has been examined experimentally and numerically [8]. The experimental study was carried over to a range of Re and Gr numbers from 1880-6220 and 14,285-714,285, respectively, while numerical calculations were performed for different ranges of same numbers. For Re numbers greater than 8000 , the Gr number had no effect on heat transfer rates and the results for unlike values of $\mathrm{Gr}$ are consistent. Heat transfer on a rotating cylinder was studied numerically [9]. The forced convection with a constant nondimensional rotational speed between 0 and 6 for Re numbers from 20 to 200 and for a fixed Pr. 
It has been found that the mean Nusselt number decreases with increasing rotational rate and increases with increasing Re.

Mixed convection heat transfer has been studied numerically in a trapezoidal cavity with a rotating heated cylinder [10]. The study concentrated on the influence of the variation of the inertia effect of the rotating cylinder on the parameters of the Re number for different $\mathrm{Gr}$ numbers ranging from 103 to 105 and for constant value of Richardson's number of 1 .

The effect of a thin heated plate located in a square cavity was analyzed numerically [11]. The effect of inclination angle and Gr number on flow and heat transfer has been investigated, it can be noticed for low Gr numbers, the variation of the inclination angle has no influence on the heat transfer. While the behavior changed for higher value of Gr number. It was observed that for each case studied there was a direction that delivered best thermal performance.

Mixed convection heat transfer has been studied a long rotating cylinder for the range of the Ri number $(0 \leq \mathrm{Ri} \leq 1)$, the rotation angle $(\propto \leq 4)$, the Re number range $1-40$ and fixed value of the Pr number [12]. It was noticed that for the particular value of rotation angle, the average $\mathrm{Nu}$ number decreased with decreasing Re number, and with increasing the angle for fixed value of $\mathrm{Ri}$ and $\mathrm{Re}$. Heat transfer using a nanofluid around a rotating cylinder with a constant nondimensional rotation rate varying from 0 to 5 was studied [13]. The study occurred at nanoparticle volume fractions range of $0-5 \%$, Re number range of $(5-40)$. It was noticed that the average $\mathrm{Nu}$ number was decreased with increasing value of the rotation rate for the fixed Re number.

The effects of a heated rotating cylinder in a cavity have been studied experimentally. The experiments were investigated for the bottom heated plate temperature ranging from $313 \mathrm{~K}$ to $333 \mathrm{~K}$. The study showed that the heat transfer inside the cavity was improved due to the rotation of inner cylinder [14]. Mixed convective nanofluid flow within a triangular cavity with a flexible sidewall under the effect of an inner rotating cylinder was analyzed numerically [15]. It was noticed that the anti-clockwise rotation of the cylinder increased the heat transfer enhancements as compared to clockwise rotation. Also, it can be observed that adding nanoparticles resulted in $49.63 \%$ enhancement of heat transfer.

The heat transfer on a rotating cylinder was investigated for varying rotation rate, $\mathrm{Re}$ and Pr numbers [16]. It was noticed that for a constant Re and Pr numbers as the rotation rate decreased, the average $\mathrm{Nu}$ number was also decreased. Mixed convection heat transfer in a trapezoidal cavity was studied numerically [17]. It can be noticed as the Ri number increased the average $\mathrm{Nu}$ number increased for all aspect ratios, and it was reduced as the rotational angle increased. The laminar flow over a rotating cylinder within an enclosure were studied numerically [18]. It was observed that the local and average values of the Nusselt number improved as Reynolds number increased for a specific angle of rotation.

\section{Physical Modeling}

\subsection{Model Presumptions and Equations}

Fig. 1 shows the description of the problem for mixed convection in a 2D inclined square cavity, of width and height $\mathrm{H}$, with the rotating cylinder, the cylinder was located at the center of the cavity. Both the vertical walls are completely insulated. The lower wall of the cavity is exposed to- uniform heat flux and the upper wall fixed at cold temperature, the rotating cylinder produces 
a forced flow. The working fluid is air, incompressible fluid and the Boussinseq's approximation is used, the density of fluid is affected by temperature changes. A constant fluid properties are assumed for all remaining thermophysical fluid properties. Moreover, a laminar flow and neglect the thermal radiation heat transfer have been assumed. The governing equations can be applied on fluid flow mixed convection inside the enclosure, in its dimensionless form [19].

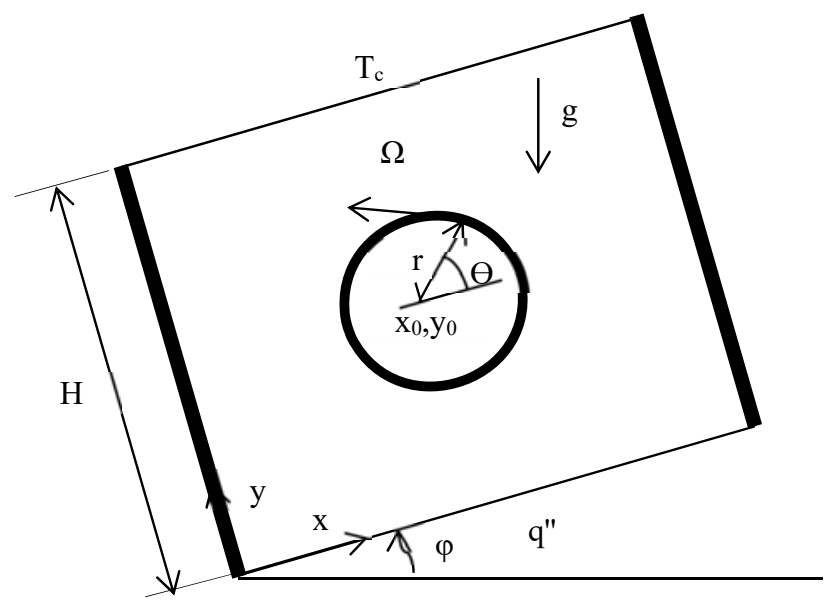

Fig. 1. The geometry and the physical model

$\frac{\partial U}{\partial X}+\frac{\partial V}{\partial Y}=0$

The equations of momentum are:

$U \frac{\partial U}{\partial X}+V \frac{\partial U}{\partial Y}=-\frac{\partial P}{\partial X}+\operatorname{Pr}\left(\frac{\partial^{2} U}{\partial X^{2}}+\frac{\partial^{2} U}{\partial Y^{2}}\right)+R a \operatorname{Pr} T \sin \varphi$

$U \frac{\partial V}{\partial X}+V \frac{\partial V}{\partial Y}=-\frac{\partial P}{\partial Y}+\operatorname{Pr}\left(\frac{\partial^{2} V}{\partial X^{2}}+\frac{\partial^{2} V}{\partial Y^{2}}\right)+\operatorname{RaPr} T g \cos \varphi$

The energy conservation equation:

$U \frac{\partial T}{\partial X}+V \frac{\partial T}{\partial Y}=\frac{\partial^{2} T}{\partial X^{2}}+\frac{\partial^{2} T}{\partial Y^{2}}$

The parameters in a dimensionless form which are used in the previous equations can be clarified as following [19]:

The space coordinates:

$(X, Y)=\frac{(x, y)}{H}$

The velocity components:

$(U, V)=\frac{(u, v)}{\alpha \mathrm{f} / H}$

The temperature: 
$\mathrm{T}=\left(\mathrm{T} *-\mathrm{T}_{\mathrm{c}}\right) /\left(\mathrm{T}_{h}-\mathrm{T}_{\mathrm{c}}\right)$

The driving pressure:

$\mathrm{P}=\left(\mathrm{p}+\rho_{\mathrm{o}} g \mathrm{~g}\right) /\left[\rho_{\text {of }}\left(\alpha_{\mathrm{f}} / \mathrm{H}\right)^{2}\right]$

And the numbers of Prandtl and Rayleigh emerge as:

$\operatorname{Pr}=v / \alpha f$

$\mathrm{Ra}=\mathrm{g} \beta\left(\mathrm{T}_{\mathrm{h}}-\mathrm{T}_{\mathrm{c}}\right) \mathrm{H}^{3} /(v \alpha)$

The definition of heat transfer coefficient in terms of the local Nusselt number $(\mathrm{Nu})$ and average Nusselt number $\left(\mathrm{Nu}_{\text {avg }}\right)$ is given as:

$N u=-\frac{\partial T}{\partial n}$

$N u_{\text {avg }}=\frac{1}{L} \int_{0}^{L} N u d L$

The dimensionless governing parameters:

$R=\frac{r}{H}$

$\Omega=\frac{\omega * \mathrm{H}^{2}}{\alpha_{\mathrm{f}}}$

For the rotating cylinder, the dimensionless formula of velocity modules are explained below:

$\mathrm{U}=-\Omega\left(\mathrm{Y}-\mathrm{Y}_{0}\right)$

$\mathrm{V}=\left(\Omega \mathrm{X}-\mathrm{X}_{0}\right)$

The absolute value of velocity at any point of the cylinder can be evaluated as:

$|V|=\sqrt{U^{2}+V^{2}}=|\Omega| R$

For the present study and dimensionless parameters:

$$
\begin{aligned}
& R i=\frac{R a P r}{4 \Omega^{2} R^{4}} \\
& \frac{\partial \psi}{\partial X}=-v \\
& \frac{\partial \psi}{\partial Y}=u
\end{aligned}
$$
1- $\frac{\partial T}{\partial Y}=1$ at bottom wall
2- $\mathrm{T}=0$
at upper wall 

3- $\frac{\partial T}{\partial Y}=0$
at vertical walls (presumed to be insulated)
4- $\frac{\partial T}{\partial Y}=0$
at the surface of the cylinder (are assumed to be insulated)

\section{Result and Discussion}

\subsection{Numerical Scheme and code Validation}

The flexPDF package used Galerkins weighted finite element remainder scheme to numerically solve the dominant Eq. (1) to Eq. (4). The values distribution of $(\partial \mathrm{U} / \partial \mathrm{X}+\partial \mathrm{V} / \partial \mathrm{Y})$ through the domain is presented in Fig.2. It is clear that the continuity equation is exactly validated.

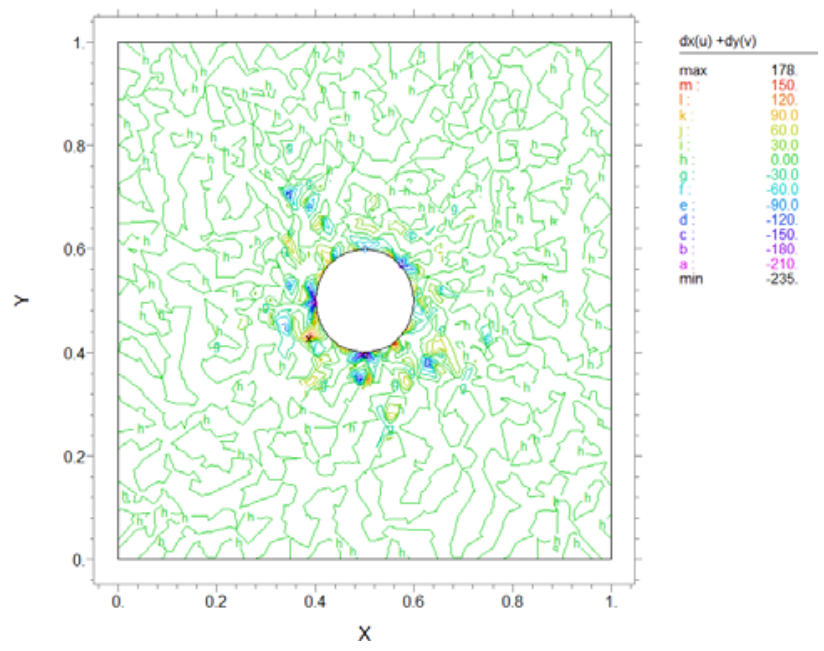

Fig. 2. Validation of continuity equation

For the developed model validation, simulations were performed to compare the mixed convection case of a heated rotating cylinder in a square cavity, as indicated in [19]. The summary of the comparison is shown in Table 1, a good agreement has been achieved by comparing the results of this work with other previous work [19]. 
Table 1. The comparison of the surface average present work Nusselt number with $\left(\mathrm{Ri}=10^{3}, \mathrm{~L} / \mathrm{R}=5, \mathrm{Pr}=0.71\right)[19]$.

\begin{tabular}{lll}
\hline \multicolumn{2}{c}{$\mathrm{Nu}_{\text {avg }}$} \\
\hline $\mathrm{Ra}$ & Present work & Liao and Lin [19] \\
\hline & & \\
$10^{4}$ & 6.44 & 6.5 \\
$10^{5}$ & 9.86 & 9.8 \\
\hline
\end{tabular}

\subsection{Flow field and Isotherms}

The effect of tilt angle is examined for $\Theta=00,300,600,900$, the flow and thermal field in the cavity in the form of streamlines (right) and isotherms (left) for $\mathrm{Ra}=104$ at $\Omega=1000$ are shown in Figure 3.

In this figure, fluid flow takes place on the space between the cylinder and the enclosure, and it consists of longitudinal cells located in the gap area at the bottom and the upper of the cylinder this due to secondary flow. At tilt angle $\Theta=0^{\circ}$, there is a big circulating cell cover all the whole cavity, and a number of small cells surrounding the great cell at the core of the cavity. Figure shows a uniform temperature distribution near the lower wall and upper wall of the enclosure, and a weak heat transfer by natural convention is take place between the walls and the fluid.

With an increase in the tilt angle of hot surface, a recirculation cell develops in the gap area at the top of the cylinder and the other at the bottom of the cylinder leading to a significant change in the streamlines structure. The streamlines are very dense, thus indicating intense fluid flow, this an expected result due to the natural convection effects and rotation effects on the fluid motion. By observing Fig. 3, it is at angle $30^{\circ}$ that flow changes its behavior from one pattern to the other. From angle $\Theta=30^{\circ}$ to $90^{\circ}$, the flow field is a strong circulation moving to upward along the hot wall and moving to downward along the cold wall, so that the vortices merge above the cylinder to form a single longitudinal vortex, also the same vortex is take place near the bottom surface of the cylinder, which is located in the core of the enclosure.

For isotherms, the figures show that as the tilt angle of the heated surface increases towards the vertical position, the direction of the convection currents tends to become parallel to the hot surface and parallel to the direction of fluid movement at the angle $\theta=90 \mathrm{o}$, where the isothermal pattern becomes thinner. There is a uniformity in the temperature distribution within the gap due to the effect of rotation, which is typical of convection problems in closed cavities. The streamlines are affected by the rotating cylinder, with increasing angular velocity the vortex pattern splits into many cells and thus forced convection becomes dominant.

The case of the anticlockwise rotating cylinder is shown in this figure. Isotherm are spread in the fluid where some thermal layers can be observed near the hot and cold walls. The significant expansion of the recalculating cell presses the flow path. Convective heat transfer associated with the cell causes a removal of heat from the heated wall. The convection heat transfer enhanced by the rotation of the cylinder plays an important role in the heat exchange between the walls of the cavity and the fluid. The heat transfer process is mainly dependent of the cylinder 
motion. The thermal field represented by the temperature distribution are uniformly around the cylinder at the low and high values of angular velocity.

\subsection{Nusselt number}

The average Nusselt number changes with angular velocity at different inclination angles at $\mathrm{Ra}=10^{4}$ is depicted in Fig. 5 .

The general outcome from the plot is that $\mathrm{Nu}$ increases with angular velocity. For rotating velocities close to zero (nearly motionless cylinder), the situation corresponding to inclination angle $\Theta=90^{\circ}$ presents the minimum average Nusselt number. Apparently, there is only a small heat transfer between the walls of the enclosure and the fluid.From the figure it can be seen that the value of the Nusselt number increases with the increase in the angular velocity. For rotating velocities close to zero (a nearly motionless cylinder), the lowest value for the average Nusselt number occurs when the heated surface is in the vertical position where the least heat exchange occurs between the gap walls and the fluid.

When the rotational speed increases, the intensity of the flow lines increases and the thermal boundary layer covers the entire area of the gap, which leads to an improvement in heat exchange and an increase in the Nusselt number. When the angle $\Theta=0^{\circ}$, the direction of the flow is perpendicular to the direction of the buoyant force, so the heat is transferred from the heated surface due to the effect of the buoyant force and with help of the rotation process which lead to an improvement in heat transfer coefficient. When the angle of inclination change from the horizontal to the vertical position, the direction of the convection currents resulting from the buoyant force gradually change until it becomes parallel to the direction of the fluid movement at $\Theta=90^{\circ}$, so the heat transfer coefficient decreases with the increase of inclination angle. 

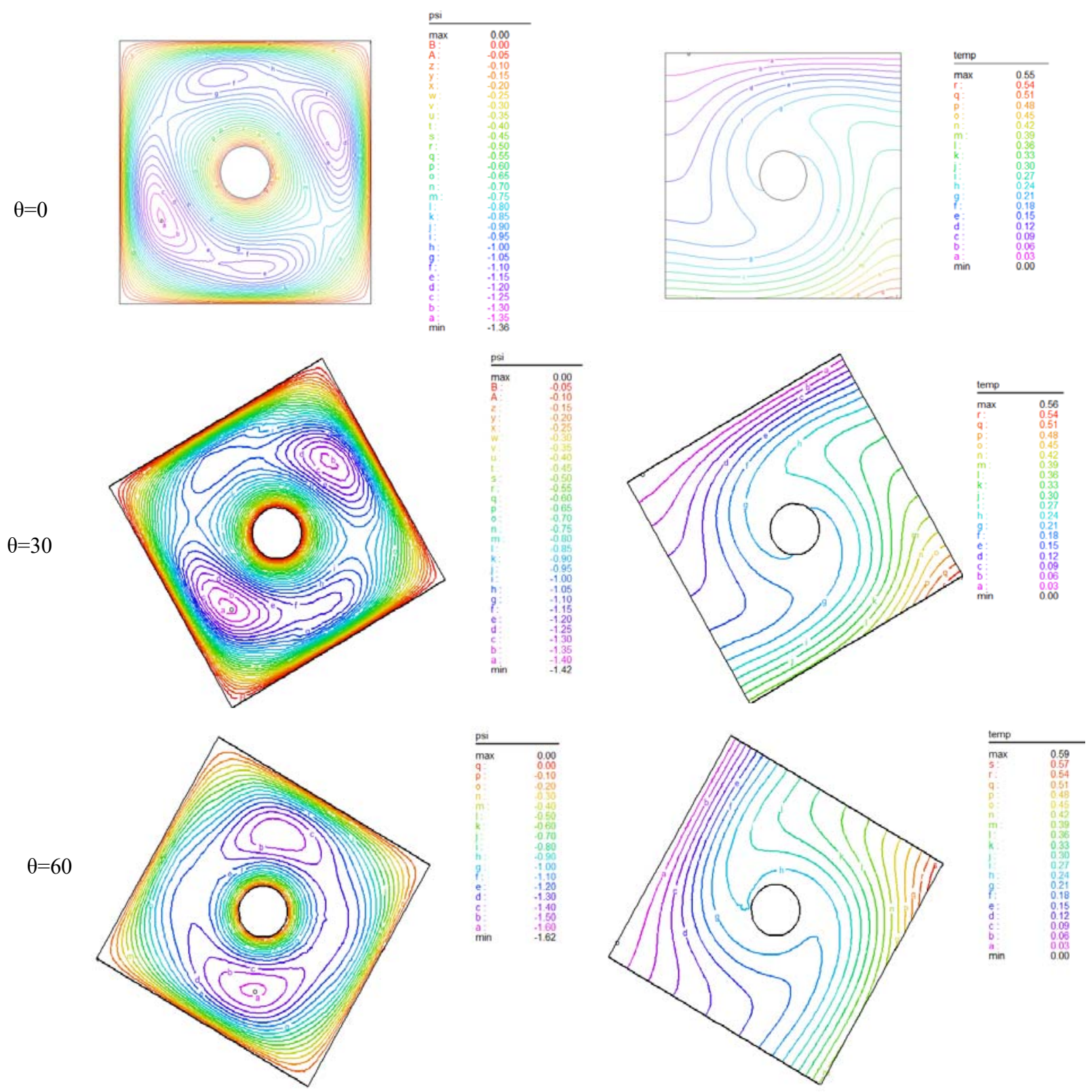

$\theta=90$
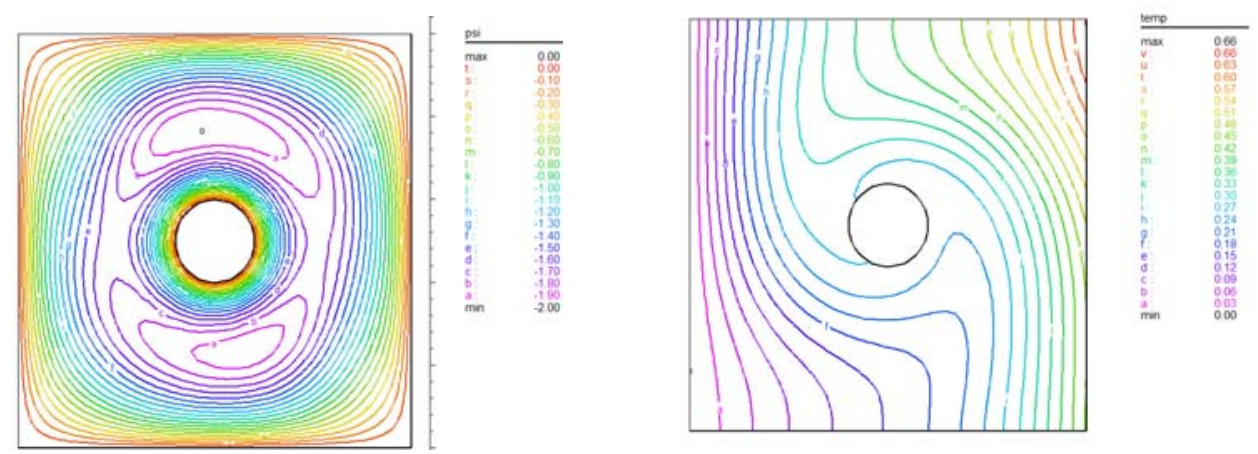

Fig .3: Isotherms and streamlines for different inclination angles at $\Omega=1000, \mathrm{Ra}=10^{4}$ 

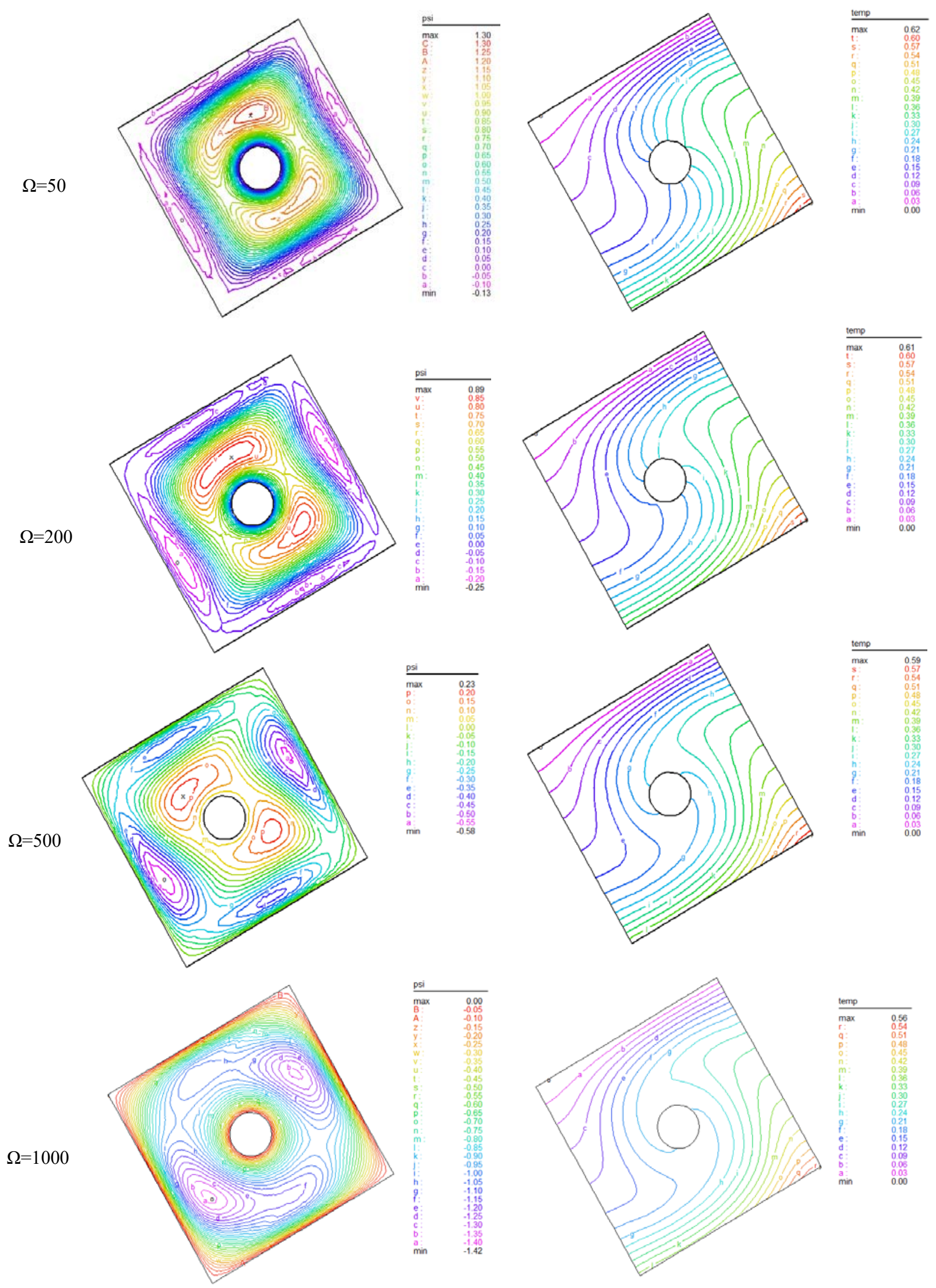

Fig.4: Streamlines and isotherms for different angular velocity at $\Theta=30^{\circ}$ and $\mathrm{Ra}=10^{4}$ 


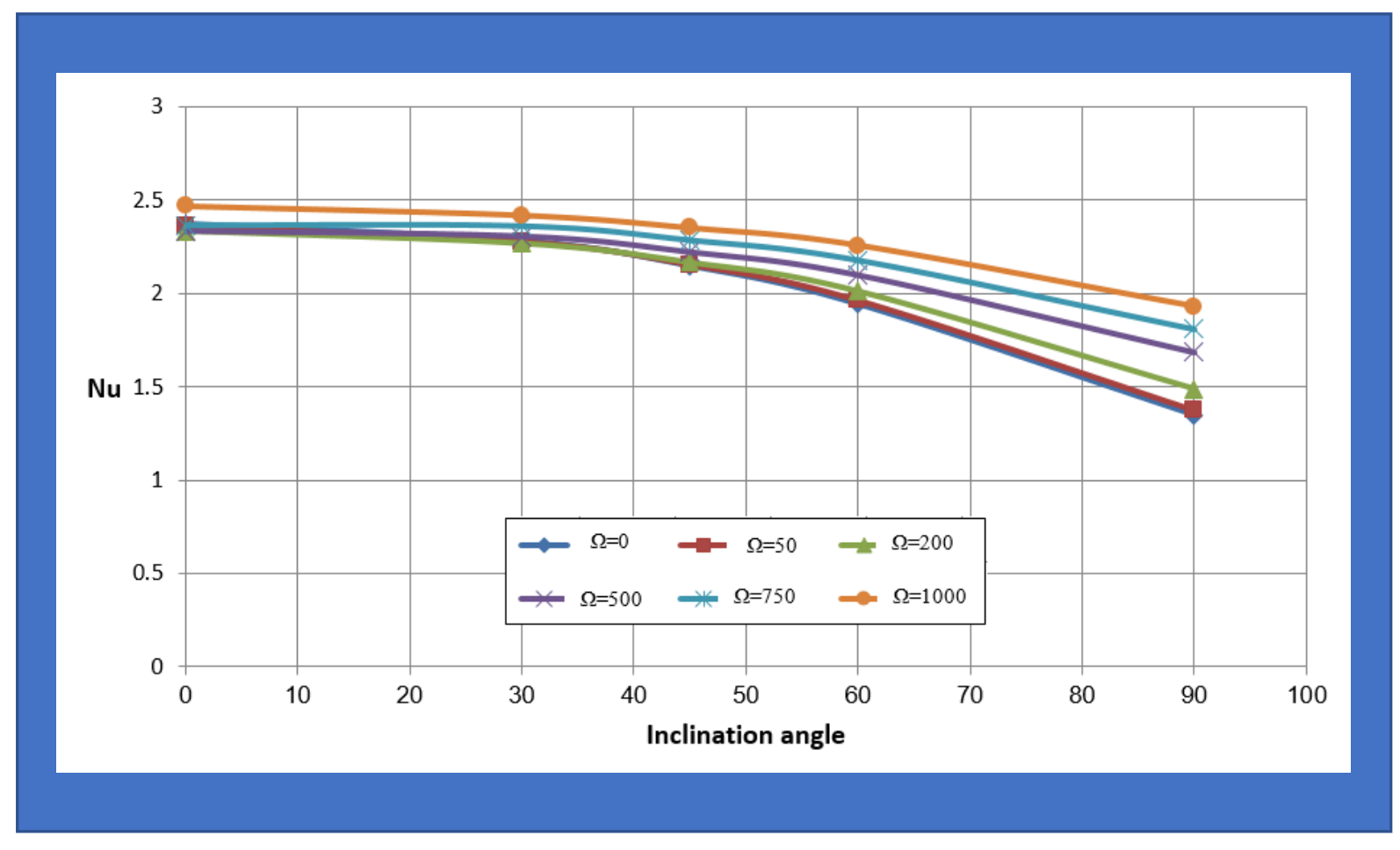

Fig. 5: The average Nusselt number changes with angular velosity at different inclination angles for $\mathrm{Ra}=10^{4}$

\section{Conclusions}

The important conclusions for mixed convective heat transfer in a square enclosure of the flow can be drawn from the numerical simulation results as:

- The effect of circulating flow by the cylinder increases the density of the isotherm lines, making it cover most area of the cavity

- When the angular velocity is increased, the results showed a distortion in the streamline distributions near the surface of the cylinder due to the strong influence of the secondary flow.

- As the inclination angle increases, the buoyancy force increases and the thermal lines become more intense.

- Streamlines are affected by the angular velocity, where the forced convection becomes dominant at high values of angular velocity.

- The rotation of the cylinder has a strong effect on the heat exchange between the walls of the gap and the fluid

- At angle $30^{\circ}$ and angle $60^{\circ}$, the effect of angular velocity on the heat transfer process is small due to the effect of the direction of the convection currents.

- The value of Nusselt number increases with the increase in angular velocity and decreases with the change of the tilt angle of the hot surface from the horizontal to the vertical position. 


\section{References}

[1] A. Barletta, S. Lazzari. (2005), 2D free convection in a porous Cavity heated by an internal circular boundary, The COMSOL Multiphysics User's Conference Stockholm. 2005.

[2]V.A.F. Costa, A.M. Raimundo. (2010), Steady mixed convection in a differentially heated square enclosure with an active rotating circular cylinder. International Journal of Heat and Mass Transfer 53 1208-1219, 2010.

[3] S. H. Hussain, Q. R. Abd-Amer. (2011) Mixed Convection Heat Transfer Flow of Air Inside a Sinusoidal Corrugated Cavity With a Heat Conducting Horizontal Circular Cylinder. Journal of Enhanced Heat Transfer, 18 (5): 433-447, 2011.

[4] F.H. Ali, (2011) Study of mixed convection in a square enclosure with a rotating circular cylinder at different vertical locations, The Iraqi Journal For Mechanical And Material Engineering, Vol.11, No.2,242-252, 2011.

[5] A. K. M. Alshara. (2012) Effect of Single or Multi Rotating Horizontal Cylinders on The Mixed Convection Heat Transfer Inside a Triangular Enclosure. Al-Qadisiya Journal for Engineering Sciences Vol. 5 No. 1. 2012.

[6] H. Xu, R. Xiao, F. Karimi, M. Yang, Y. Zhang, (2014), Numerical study of double diffusive mixed convection around a heated cylinder in an enclosure, International Journal of Thermal Sciences 78, $169 \mathrm{e} 181,2014$.

[7] Everts, M, Ebrahim R., Kruger, J.P., Miles, Sharifpur M. and Meyer, J.P, (2014) Turblent flow a Cross a Rotating Cylinder with Surface Roughness, HEFAT, 10th International Conference on Heat Transfer, Fluid Mechanics and Thermodynamics. Orlando, Florida, 2014.

[8] R.I.(2014) Elghnam Experimental and numerical investigation of heat transfer from a heated horizontal cylinder rotating in still air around its axis, Ain Shams Engineering Journal 5, 177-185, 2014.

[9] R. Bouakkaz1, K. Talbi1, M. Ouazzazi, Y. Khelili1 and F. Salhi. (2015) Effect of Rotation Rates on the Laminar Flow and Heat Transfer Past a Circular Cylinder. Vol. 32, No. 02, pp. 519 - 529, April - June, 2015.

[10] M. Khan, A. A. Khanb, M. N. Hasan. (2016) Numerical study of mixed convection heat transfer from a rotating cylinder inside a trapezoidal enclosure. International Conference on Mechanical Engineering, 2016.

[11] N. Hamici, D. Sadaoul. 2017 Numerical Study of Mixed Convection and Thermal Radation in a Square Cavity with an Inside Inclined Heater. Lille, 28 Août au 1er Septembre, 2017.

[12] K.Yahiaoui1, D. Nehari, B.Draoui, (2017), The Investigation of the Mixed Convection from a Confined Rotating Circular Cylinder, Periodica Polytechnica Mechanical Engineering, 61(3), pp. 161-172, 2017.

[13] R. Bouakkaza, F. Salhib, Y. Khelilic, M. Quazzazid, K. Talbi, (2017), Unconfined laminar nanofluid flow and heat transfer around a rotating circular cylinder in the steady regime, archives of thermodynamics,Vol. 38, No. 2, 3-20, 2017.

[14] M. F. Ejaz, S. Manzoor, (2018) Experimental Investgation of Heat Transfer in a Vertical Annulus with a Bottom Heated Rotating Inner Cylinder, International Journal of Heat and Technology, Vol, 36, No.2, 2018.

[15] F. Selimefendigil, Hakan F. Oztop, Ali. J. Chamkha. (2019) Analysis of mixed convection and entropy generation of nanofluid filled triangular enclosure with a flexible sidewall under the influence of a rotating cylinder. Journal of Thermal Analysis and Calorimetry, 135:911-923, 2019.

[16] V. Sharma, A. K. Dhimman, Heat Transfer from a Rotating Circular Cylinder in the Steady Regim: Effects of Prandtl Number.

[17] M. A. H. Mamun, T. R. Tanim,M. M. Rahman, R. Saidur and Shuichi Nagata, (2011) Analysis of Mixed Convection in a Lid Driven Trapezoidal Cavity, Convection and Conduction Heat Transfer,

[18] A. Anguraj and J. Palraj, Numerical study of fluid flow and heat transfer in a backward facing step with a rotating cylinder, Department of Mathematics, PSG College of Arts and Science, Tamil Nadu, INDIA. 
[19] C. C. Liao and Lin. C. A, Mixed convection of a heated rotating cylinder in a square enclosure, Department of Power Mechanical Engineering, National Tsing Hua University, Hsinchu 30013, Taiw. International Journal of Heat and Mass Transfer 72 9-22, 2014. 